\title{
An Investigation into the Measurement and Prediction of Mechanical Stiffness of Lower-limb Prostheses used for Running
}

Two energy return prosthesis are subjected to three different statically applied loading methods. This initial study proposes that statically applied loading to a sport prosthesis using several controlled methods were statistically robust enough to derive a mechanical stiffness value. However, any predicted stiffness is drawn into question when allowing any movement of the distal end. This uncertainty will make any evaluation or prescription of lower-limb prosthesis technology based upon their stiffness incorrect. In addition, the peak calculated stiffness at the expected bodyweight induced ground impact load of a runner is judged the most representative assessment method.

This study attempts to build on previous research advocating the need to monitor the performance of prosthesis lower-limb technology in disability sport.

Keywords: amputee; sprinting; stiffness; prosthesis

Practitioner Summary: This paper extends previous research regarding the fairness of prosthetics technology used in running with a lower-limb amputation. It pilots a quantitative assessment of high activity prosthetics technology and ultimately demonstrates how incorrect assessment can lead to incorrect specification of running prosthesis for elite level sport.

\section{Introduction}

Lower-limb energy storage and return prostheses (ESRP) are currently used in competitive running by athletes with an amputation ranging from the $100 \mathrm{~m}$ upto longer events such as the marathon. Whilst the design requirements for such endeavours may 
vary in terms of their geometry and response (Nolan, 2008), the general principle of their operation is typically the same. This is effectively one of a spring manufactured from a composite and when running, the mass of the athlete dynamically compresses the prosthesis. During the gait cycle, the prosthesis will store and then return a percentage of the energy which was applied to it. The prosthesis essentially restores some of the ability that would conventionally be supplied by a biological lower-limb.

Whilst research has argued if an athlete with a double below knee amputation may be physiologically advantaged when using this technology (Bruggemann et al. 2008) or not (Grabowski et al. 2009), it has been shown that the actual mechanical energy return produced by these prostheses themselves is far below that of the biological limb they replace. The energy return has been shown to be as high as $95 \%$ but this is far less than the ankle which has been shown to generate $241 \%$ energy return (Nolan, 2008). There is also no definitive research to date whether a double (bi-lateral) or single (uni-lateral) amputees should be considered separately with respect to any performance enhancement. Whilst it seems that the endeavour should therefore fall on engineers or prosthetists to maximise the performance of such technology, concern has been raised about athlete to athlete parity when allowing such technology in international disability sprinting with a lower-limb unilateral amputation (Dyer et al. 2011). In addition, prosthetics ‘equipment' should be monitored and effectively regulated to ensure a fair sport in the future (Dyer et al. 2010).

For any sport's governing body or stakeholder to provide the ability to measure the performance of such technology in the future, the key performance indicators of short distance sprinting should be established. The general key biomechanical performance indicators of maximal running speed have been proposed as ground reaction force (Weyand, 2000), short ground contact time (Nummela et al. 2007), stride 
length (Cronin et al. 2007), frequency (Paruzel-Dyja et al. 2006; Babic et al. 2007) and limb stiffness (Bret et al. 2002). Of these observations, only stiffness is a mechanical characteristic that relates specifically to the lower-limb. Biological limb stiffness has been shown to correlate strongly with maximal running (Chelly and Denis 2001, Bret et al. 2002) or at the very least, that as a bi-product, lower-limb stiffness increases with increasing running speed (Brughelli and Cronin 2008). Interestingly, a prosthetic augmented lower limb has indicated a reduction in lower-limb stiffness (McGowan, 2012) but this has seen extremely limited subject sample numbers to date. Biological limb stiffness is provided predominantly from the knee joint and to a lesser extent as a constant from the ankle (Brughelli and Cronin 2008). However, when fatigue of the biological limb takes place (such as towards the end of a race), despite the net lowerlimb stiffness remaining unchanged, it has been proposed that a compensatory mechanism via a greater contribution from the ankle can take place (Padua et al. 2006).

In addition, it has been shown that limb stiffness is independent of leg strength in $100 \mathrm{~m}$ sprinting which makes it ideal to compare the mechanical limb to biological limb performance (Bret et al. 2002). It should be noted though, that if stiffness has been proposed to remain constant based upon the demands placed upon it, the stiffness of the limb does change through the gait cycle due to the loading and unloading of the limb (Bret et al. 2002). This means that unlike the current passive prosthesis used in Paralympic running, the biological region it is substituting is a fully active, dynamic limb, constantly changing its behavioural properties. However, dynamically controlled prostheses which could simulate this effect are not currently legal for competition in international athletics.

An extensive review of running stiffness measurement methods was undertaken by Brughelli and Cronin (2008). In this, a clear distinction is made between the 
assessment of leg stiffness, vertical stiffness, and then other more focused subsets such as joint or tendon stiffness. Vertical stiffness can be obtained by using force plates or pressure sensors to record impact forces coupled with recording changes in vertical displacement using high speed camera's with reflective markers. The prosthesis has been proposed to be compared mechanically to the same biological region it has replaced (Dyer et al. 2011) so leg stiffness is more relevant to the prosthesis directly than vertical stiffness of the leg as a whole.

Few studies have evaluated the specific key performance indicators of unilateral lower-limb amputee sprinting although several have noted physical impedance of distinctive features in both trans-tibial (below knee) and trans-femoral (above knee) amputee runners such as limb to limb asymmetry (Burkett at al. 2003), energy transfer compensation (Czerniecki et al. 1996) and joint moment differences (Buckley, 2000). The key performance indicators in able-bodied subjects of lower-limb stiffness and ground reaction force have not seen evidence to date as being any less critical for lowerlimb amputee sprinting. Indeed, improved lower-limb stiffness has been demonstrated to improve biological to mechanical limb symmetry in amputees (Lechler, 2005).

The current specification of ESRP are typically based upon the bodyweight of the proposed user (Nolan, 2008). Each of the manufacturers' range of ESRP will have a different response in their inherent stiffness depending on which product is selected. In some cases, manufacturers determine this stiffness through statically applied loads from strength testing machines. In the case of prosthesis used for high levels of activity such as performance sprinting, no standardised test currently exists. Formal existing test protocol standards such as ISO 10328:2006 deal with static loading conditions and define both the load speed and magnitude, but these relate primarily to the stance phase 
of walking and the use of clinical prosthesis and not those used in high performance sport.

Whilst sport with a disability may have initially been about participation (Gold and Gold 2008), successful athletes can be as rewarded as their able-bodied counterparts. As a result, the sport offers incentives and rewards for the very best athletes. There have been cases of such technologies fairness being questioned when used in able-bodied sport such as with the case of Oscar Pistorius in 2008 (Hilvoorde and Landeweed, 2008). Therefore, if a sport's governing body wishes to monitor prosthesis technology to maintain fairness between its competitors (such as at the Paralympics), the ability to feasibly measure its key performance indicators should be investigated.

This study will initiate and pilot this process by evaluating the stiffness of ESRP's when using three different statically applied loading methods. Previous research has evaluated the use of such methods and found that such techniques have limitations (Dyer et al. 2013). However, this study will extend this by attempting to predict the stiffness of prosthetic blades at higher loads by using different trend methods. This study continue to focus on developing methods to assess the performance of lower-limb performance technology in isolation as philosophically advocated in the Dyer et al. (2011) study.

\section{Methodology}

Two ‘Elite Blade’ composite ESRP’s (Chas A Blatchford \& Sons Ltd, Basingstoke, UK) were used in this study as typical examples of high activity prostheses. The Elite Blade is a composite prosthetic foot that is designed for high levels of activity such as jogging and running with its specification dependant on the mass of the amputee that 
uses it (Figure 1). This is the same technology in principle as those used in elite competition. They have a split toe design which sometimes utilises an attached 'heel'. This heel component was omitted from these studies so as to focus solely on the carbon 'blade' itself. The lengths of the prostheses are approximately $405 \mathrm{~mm}$ from end to tip. The thickness of the composite material is approximately $10 \mathrm{~mm}$ along the length of their shanks but then progressively tapers down to 3mm at their distal ends.

Neither of the prostheses was modified in any way and appeared visually similar in their design. They were numbered prosthesis 1 and 2 . Their exact performance specification was not known prior to these experiments.

(Insert figure 1)

The prostheses were vertically compressed in a fixed, inverted position using a Testometric strength testing machine (Testometric Company Ltd, Lancashire, UK).

Taking the length of the supplied prostheses into account, both of them were mounted to an aluminium fixing block which aligned the prostheses shank at a 60 degree angle from a horizontal plane. This selected angle ensured correct alignment creating a theoretical centreline that would run from the distal end of the prostheses through the midpoint of the fixing bolts that would normally attach to the prosthesis socket. An example image of the experiment set up is shown in Figure 2.

(Insert figure 2)

Both prostheses were loaded in compression 10 separate times up to a load of 1500$2000 \mathrm{~N}$ and applied at a rate of $50 \mathrm{~mm}$ per minute. Due to the lateral shear forces 
imposed when loading the prostheses in test trials prior to these experiments, these were the safest maximum loads it was felt to impose in these experiments. Each test took approximately 1 minute to complete.

The three different prostheses compression static loading techniques illustrated in Figure 3 are described as below:

(1) Fixed at the prostheses distal end (FDE). The distal end of the prosthesis butts against a ledge which prevents it from sliding when compressed.

(2) Partial slide then fixed (PSF). The prosthesis is allowed to slide $28 \mathrm{~mm}$ before the distal end butts against a ledge preventing further slide when compressed.

(3) Unfixed distal end (UDE). The distal end of the prosthesis is unfixed and can slide freely under the load cell platen when compressed.

(Insert figure 3)

The Testometric platen (which contacts with the prosthesis) was made of nylon and was machined to provide the three separate conditions tested. Prosthesis 2 was not tested under condition three due to its arrival later in the testing phase after the platen had already been modified for conditions one \& two.

The experiment was pilot tested prior to this study to ensure repeatability of both the measurement equipment and protocol.

Mechanical stiffness was calculated in this instance as load divided by deflection. This is calculated as average load divided by average deflection of the entire load cycle sample measured. In addition, the stiffness obtained from the peak load and resultant deflection at the maximum applied load is also calculated for comparison. 
The Coefficient of Variation (CV) is used to calculate data's variability in this study. The CV has been defined as a measure of absolute consistency when evaluating a series of results (Stalbom et al. 2007) and is calculated as the Standard Deviation divided by the Mean Average and then multiplied by 100. The lowest possible percentage is most desirable.

Next, it is investigated to see if a limited subset of load and deflection data could be used to accurately predict the stiffness response of higher loads. This would be corroborated with an additional load and resultant deflection test to the targeted higher load. This is useful as it was proposed in this papers introduction that lower-limb stiffness changes based on running speed (Brughelli and Cronin 2008) and in addition, it has been proposed that sprinting witnesses bodyweight impacts of 4-5 times bodyweight (Mero et al. 1992). Therefore a successful method of prediction would allow prosthesis stiffness to be calculated for greater loads than those investigated in this study or for different running events based on a smaller subset of data.

The predictions will use prosthetic loading data from experiment 1 but be corroborated with a second data set loaded to the higher load of $3500 \mathrm{~N}$. The experiment 1 data will have two trend lines extended from the first experiment data of the FDE method to help predict the response. These are:

- $\quad$ A $2^{\text {nd }}$ order polynomial line of the entire load and deflection graph trace.

- A linear line extended from a $450 \mathrm{~N}$ loading sample taken at the end of the load and deflection graph trace.

\section{Results of Load-Deflection Data}


When conducting the designated number of loadings of each method using the two prostheses, the following data was produced for comparison and is shown in Table 1.

(Insert Table 1)

With both prostheses, the FDE method demonstrated the highest recorded average stiffness.

The UDE method only used on prostheses 1 highlights a vastly inferior recorded average stiffness. However, in this specific case, the distal end is not fixed meaning the prosthesis is constantly slipping or 'arching through' as it is compressed. The recorded deflection is therefore a relative rather than a true deflection. In addition, due to the controlled slippage, the prostheses total length is effectively shortening as it is being compressed. Due to the amount of bend evident, it was decided for safety reasons to cease loading at $1500 \mathrm{~N}$.

Typical behaviour of all the tests of prostheses 1 and 2 are shown in figure 4-8

(Insert Figure 4-8)

\section{Results: Predictive Performance of a 3500N Loading}

One prosthesis (prosthesis 2, FDE method) is used to ascertain whether a predictive stiffness can be generated from a smaller load and deflection data set that stops at a lesser amount $(2000 \mathrm{~N})$. By then applying both a $2^{\text {nd }}$ order polynomial trend line and a linear trend line to the final 450N 'linear like' sample of section 3's data, the following result from one of the tests can be seen in figure 9 . 
(Insert Figure 9)

The predictive stiffness values at 3500N were calculated by rearranging the polynomial and linear line trend line calculations displayed inside the graphs in figure 9. This was achieved by determining the resultant deflection when $3500 \mathrm{~N}$ is applied. The mechanical stiffness was recalculated and then compared to new actual loading cycles of prosthesis 2 performed upto $3500 \mathrm{~N}$ of load. This data can be compared in table 2 below.

(Insert Table 2)

\section{Discussion}

Both prosthesis 1 and 2 produced different levels of stiffness to each other. This only highlights how composite manufacture can be altered by changing parameters such as cloth lay-up, fibre orientation or resin application to change the mechanical properties of a prosthesis - despite looking physically identical.

Despite some differences between the three measuring methods prostheses stiffness, all experiments each generated extremely low coefficient of variation of the data in a $0.2-1.1 \%$ range. This value is extremely low and suggests that each experiments data was stable and repeatable. It is also suggested that less than 10 tests are sufficient for each experiment test method. This low level of data variation did include the PSF and UDE methods that had incorporated intentional slippage of the distal end. Whilst the boundary conditions of each method are slightly different, the compression and slide characteristics of the methods are clearly stable. 
The highest recorded stiffness with both prosthesis 1 and 2 occurred when using the FDE method. This is likely due to both the UDE and PSF methods having controlled slippage of the distal end causing a relative rather than an actual displacement to be recorded by the loading machine. It is possible that such slippage of the PSF and UDE methods may effectively shorten the spring length causing further measurement inaccuracy.

All prostheses and their assessment methods produced non-linear behaviour and this characteristic has also been witnessed in other studies (Jaarsveld et al. 1990). The design of the ESRP's sees a material thickness change in the material lay-up of the composite which gets thinner the closer it is to the distal end. The proportion and degree of this change in thickness will likely vary from prosthesis to prosthesis based upon its model, prescription and supplier. Under load, the 'foot' and 'ankle' zones of the prosthesis with a tapered thickness will compress first, then causing an initial non-linear response. The increased (yet uniform) thickness of the shank/calf related areas in the prosthesis used here will create an increase in stiffness and will therefore produce a more linear load/deflection relationship. Taking this into account, whilst the PSF and FDE are different at the initial point of their graph trace due to the controlled slippage, the stiffness towards the top of the graph trace should hypothetically be identical. At a loading of $1950 \mathrm{~N}$, the prosthesis 1 FDE and the PSF methods had stiffness's of $60 \mathrm{~N} / \mathrm{mm}$ and $58 \mathrm{~N} / \mathrm{mm}$ respectively - a difference of 3.3\%. Prosthesis 2 had FDE and PSF stiffness's of 48 and 42 - a difference of 12.5\%. This demonstrates that whilst the prosthesis performance itself is repeatable, the change in methods produces a significant enough change in the experiments boundary conditions not to make the data referenceable between methods. With this in mind, the FDE method is recommended for use in the future because its stiffness was both higher than the PSF method coupled 
with the knowledge that its distal end was fixed and therefore likely a more accurate representation of mechanical performance. It could be argued however, that measuring stiffness by fixing the prosthesis at the distal end is not representative of it in actual use as the ground reaction strike point will likely not be at the absolute distal end. This would also be affected by changes in race length and characteristics such as running round the bend and athlete fatigue. However, because this strike point would be different between all runners, an alternative approach is to identify a standardised point along the prosthesis length that correlates to the strike point of the human foot. Some further research is warranted in this area but the relatively small population sizes of current sprinters with an amputation will make such a study difficult. Alternative approaches to technology assessment have been proposed to be as much a philosophical dilemma as those grounded in empirical science (Dyer et al. 2010).

The UDE method used with prosthesis 1 demonstrated massively different stiffness behaviour to the other two methods. It only produced a relative stiffness of approximately $56 \%$ of the fixed distal end methods. Its graph trace is more linear than both the PSF and FDE methods but this is likely due to both its spring length and recorded deflection both changing at the same time as it is compressed. The UDE method will likely lead to an underestimation of the prosthesis performance or a misprescription of such technology to athletes. An unfixed distal end when measuring stiffness of a prosthesis is not recommended.

Previous research has proposed that technology monitoring of lower-limb prosthesis could be achieved through limb-to-limb stiffness comparison (Dyer et al. 2011). With this in mind, there is debate as to whether a limb’s stiffness should be assessed by comparing its average stiffness or its stiffness at the maximum load expected when sprinting. Some studies have advocated the mechanical stiffness of a 
prosthesis by taking the mean average of the entire load/deflection data (Jaarsveld et al. 1990). 100m sprinting is a running event characterised by the highest levels of lowerlimb stiffness correlating to performance (Chelly and Denis, 2001), and the data here demonstrates that the non-linearity of ESRP cause a large disparity between the mean average and peak stiffness of the prosthesis under load. It is shown in this study to be as great as 93\% when using the PSF method. In addition, the $100 \mathrm{~m}$ has been proposed to comprise 3 specific performance phases of high acceleration, acceleration to maximal running speed and maintenance of maximal running speed (Johnson and Buckley, 2001). This suggests that an average stiffness would be the suitable, holistic approach to take the varying phases into account. However, the maximal speed phases are likely far larger as a percentage of the overall 100m race compared to the lower load efforts plus lower speeds have been demonstrated not to cause reductions in ankle joint stiffness (Arampatzis et el. 1999). As a result, if prosthesis should be judged functionally against the area it replaces (Dyer et al. 2011) and the ankle stiffness does not therefore change, the peak stiffness would be more representative of a larger percentage of the limbs actual experience in the $100 \mathrm{~m}$ event.

The predictive data attempted to ascertain whether when taking a smaller, lower load sample of load and deflection data, whether larger loads could be predicted. Theoretically, this should allow the ability to predict the mechanical stiffness of prosthesis of sprinters of different bodyweights. In this case the initial 1950N maximum load was increased by $45 \%$ to $3500 \mathrm{~N}$ (or roughly 4.4 times the bodyweight of a $80 \mathrm{Kg}$ sprinter). The $2^{\text {nd }}$ order polynomial trend line of the whole trace slightly overestimated the $3500 \mathrm{~N}$ load stiffness. It is felt that this is because the latter section of the graph becomes increasingly linear once the thinner, tapered 'foot' area of the prosthesis has maximised its compression and the thicker, constant thickness upper shank of the 
prosthesis begins to deflect under load. However when taking the upper, more linear section of the load deflection graph and then extending it to a loading of $3500 \mathrm{~N}$, the obtained stiffness from the mechanical testing and corroborated data was $45 \mathrm{~N} / \mathrm{mm}$ and $48 \mathrm{~N} / \mathrm{mm}$ respectively. The predicted performance was a stiffness of $49 \mathrm{~N} / \mathrm{mm}$ which is much closer to the actual performance. The typical graph trace shapes here were initially very non-linear which then becomes progressively linear at higher loads. This makes the sole use of either a linear or a polynomial trend line complicated. The best method would be to take the highest load and deflection data graph trace available and then apply a linear line to that aspect to predict higher load stiffness.

\section{Conclusions}

This study attempted to extend from previous research a call to identify methods of assessing lower-limb prosthesis technology used in disability sport. It began this pursuit by assessing three different techniques of statically applied loading techniques to measure lower-limb prosthesis stiffness. The prosthesis tested produced non-linear stiffness characteristics.

This study proposes that statically applied loading to a sport prosthesis using several controlled methods are statistically robust enough to derive a mechanical stiffness value but that the calculated stiffness magnitude itself is drawn into question when allowing any movement of the prosthesis distal end. Fixing an ESRP at both ends with no slippage allows a repeatable measurement of mechanical stiffness. Measuring the prosthesis stiffness by fixing the distal end with no slippage is the most easily repeatable method between prostheses designs unless another standardised point of ground foot strike contact can be identified. 
In lieu of a full range of stiffness data being available for a particular prosthesis, predictive stiffness at higher loads can also be accurately calculated by taking a latter, linear-like sample from lower load and deflection data once the main proportion of nonlinearity has taken place.

\section{Acknowledgements}

The prostheses used in this study were kindly donated by Chas A Blatchford \& Sons Ltd. (Basingstoke, UK).

\section{References}

Arampatzis, A., Bruggemann, G. \& Metzler, V. (1999). The Effect of Speed on Leg Stiffness and Joint Kinetics in Human Running. Journal of Biomechanics, 32, 13491353.

Babic, V., Harasin, D. \& Dizdar, D. (2007). Relations of the Variables of Power and Morphological Characteristics to the Kinematic Indicators of Maximal Speed Running. Kinesiology, 39, 1:28-39.

Bret, C., Rahmani, A., Dufour, A., Messonnier, L. \& Lacour, J. (2002). Leg Strength and Stiffness as Ability Factors in 100m Sprint Running. Journal of Sports Medicine and Physical Fitness, 42, 274-281.

Brughelli, M. \& Cronin, J. (2008). A Review of Research on the Mechanical Stiffness in Running and Jumping: Methodology and Implications. Scand J Med Sci Sports, 18, 417-426.

Bruggemann, G., Arampatzis, A., Emrich, F. \& Potthast, W. (2008). Biomechanics of Double Transtibial Amputee Using Dedicated Sprinting Prostheses. Sports Technology, $1,220-227$. 
Buckley, J. (2000). Biomechanical Adaptations of Transtibial Amputee Sprinting in Athletes Using Dedicated Prostheses. Clinical Biomechanics, 15, 352-358.

Burkett, B., Smeathers, J. \& Barker, T. (2003). Walking and Running Inter-limb Asymmetry for Paralympic Trans-femoral Amputees, a Biomechanical Analysis. Prosthetics and Orthotics International, 27, 36-47.

Chelly, S. \& Denis, C. (2001). Leg Power and Hopping Stiffness: Relationship with Sprint Running Performance. Medicine \& Science in Sports \& Exercise, 326-333. Cronin, J., Ogden, T., Lawton, T. \& Brughelli, M. (2007). Does Increasing Maximal Strength Improve Sprint Running Performance ?. National Strength and Conditioning Association, 29, 86-95.

Czerniecki, J., Gitter, A. \& Beck, J. (1996). Energy Transfer Mechanisms as a Compensatory Strategy in Below Knee Amputee Runners. Journal of Biomechanics, 29, 717-722.

Dyer, B., Sewell, P. \& Noroozi, S. (2013). How should we assess the mechanical properties of lower-limb prosthesis technology used in elite sport ? - An initial investigation, Journal of Biomedical Science \& Engineering, 6, (in press).

The Design of Lower-Limb Sports Prostheses: Fair Inclusion in Disability Sport. Disability and Society 25, 593-602.

Dyer, B., Redwood, S., Noroozi, S. \& Sewell, P. (2011).The Fair Use of Lower-limb Running Prostheses. Adapted Physical Activity Quarterly, 28, 16-26.

Dyer, B., Noroozi, S., Sewell, P. \& Redwood, S. (2010). The Design of Lower-Limb Sports Prostheses: Fair Inclusion in Disability Sport. Disability and Society 25, 593602.

Gold, J. \& Gold, M. (2008). Access for all: the rise of the Paralympic Games. The Journal of the Royal Society for the Promotion of Health, 127, 133-141. 
Grabowski, A., McGowan, C., McDermott, W., Beale, M., Kram, R. \& Herr, H. (2009). Running-specific Prostheses Limit Ground-force During Sprinting. Biology Letters, 6, 201-204.

Hilvoorde, I. \& Landeweed, L. (2008). Disability or Extraordinary Talent - Francesco Lentini (Three Legs) Versus Oscar Pistorius (No Legs). Sport, Ethics and Philosophy, 2, 97-111.

ISO. (2006). Structural Testing of Lower-limb Prostheses - Requirements and Test Methods. 10328:2006.

Jaarsfeld, H., Grootenboer, H., Vries, J. \& Koopman, H. (1990). Stiffness and Hysteresis Properties of Some Prosthetic Feet. Prosthetics and Orthotics International, $14,117-124$.

Johnson, M. \& Buckley, J. (2001). Muscle Power Patterns in the Mid-acceleration Phase of Sprinting. Journal of Sports Sciences, 19, 263-272.

Padua, D., Arnold, B., Perrin, D., Gansneder, B., Carcia, C. \& Granata, K. (2006). Fatigue, Vertical Leg Stiffness, and Stiffness Control Strategies in Males and Females. Journal of Athletic Training, 41, 294-304.

Paruzel-Dyja, M., Walaszczyk, A. \& Iskra, J. (2006). Elite Male and Female Sprinters Body Build, Stride Length and Stride Frequency. Studies in Physical Culture and Tourism, 13, 33-37.

Lechler, K. (2005). Lower-limb Prosthetics - Design Improvements of a Prosthetic Spring Foot. Retrieved October 3 2005, from www.oandp.org/publications/jop/2005 Nummela, A., Keranen, T. \& Mikkelsson, L. (2007). Factors Relating to Top Running Speed and Economy. International Journal of Sports Medicine. 28, 655-661. 
McGowan, C., Grabowski, A., McDermott, W., Herr, H. \& Kram, R. (2012). Leg

Stiffness of Sprinters Using Running-specific Prostheses. Journal of the Royal Society. Interface, 9, 1975-1982.

Mero, A., Komi, P. \& Gregor, R. (1992). Biomechanics of Sprint Running. Sports Medicine, 13, 376-392.

Nolan, L. (2008). Carbon Fibre Prostheses and Running in Amputees: A Review. Foot and Ankle Surgery, 14, 125-129.

Stalbom M., Holm, D., Cronin, J. \& Keogh, J. (2007). Reliability of Kinematic and Kinetics Associated with Horizontal Single Leg Drop Jump Assessment: A Brief Report. Journal of Sports Science and Medicine, 6, 261-264.

Weyand, P., Sternlight, D., Bellizzi, M. \& Wright, S. (2000). Faster Top Running Speeds are achieved with Greater Ground Forces Not More Rapid Leg Movements. Journal of Applied Physiology, 89, 1991-1999. 